\title{
Evaluation of complete blood count parameters in the first trimester: an early indicator of miscarriage?
}

\author{
İbrahim Kale ${ }^{1 *}$, Çağlar Helvacioğlu ${ }^{1}$, Tuğba Erel Muğurtay ${ }^{1}$ \\ ${ }^{1}$ UMRANIYE EDUCATION AND RESEARCH HOSPITAL, DEPARTMENT OF OBSTETRICS AND GYNECOLOGY, ISTANBUL, TURKEY
}

\section{ABSTRACT}

Objective. Investigation of the relationship between the first trimester complete blood count parameters and miscarriage. Materials and Methods. The study group consisted of 39 patients who admitted to the hospital with the diagnosis of abortus imminens between January 2016 and December 2019 , and whose pregnancy ended as miscarriage before the 20th week. The control group consisted of 200 randomly selected healthy pregnant women, with follow-up and delivery in our hospital. Patient information was obtained retrospectively from the hospital records. Results. Age and BMI of both groups were similar. There was no significant difference between the two groups in terms of basophil, neutrophil, eosinophil, lymphocyte, monocyte, platelets, RBC, hemoglobin, hematocrit, MCV, MCH, MCHC, RDW, MPV, Pct and PDW, while leukocyte and neutrophil counts was statistically significantly higher in the miscarriage group $(p=0,002, p=0,001$, respectively). NLR was statistically higher in the miscarriage group $(\mathrm{p}=0,005)$, PLR was also higher in the miscarriage group, but this did not reach statistical significance $(\mathrm{p}=0,056)$. Both groups were similar in terms of MLR. Conclusions. High NLR levels obtained from the hemogram results in the first trimester seems to be associated with miscarriage. NLR can be an easy, cheap and useful tool for predicting pregnancy prognosis in patients with abortus imminens.

\section{Introduction}

Vaginal bleeding occurs in the first trimester in about $25 \%$ of all pregnancies. The differential diagnosis includes bleeding due to the abortus imminens, early pregnancy loss, ectopic pregnancy and non-obstetric causes [1]. The diagnosis of abortus imminens in a patient with vaginal bleeding can be made by observing the embryo with a heartbeat in the gestational sac in the uterus with closed cervix, in an early pregnancy during an ultrasound examination [2].

The reason for bleeding in abortus imminens is often associated with the decidual veins on the maternofetal unit. After a viable fetus is detected in ultrasonography, the rate of early pregnancy loss is approximately $11 \%$, but the risk of pregnancy loss increases if there is an area of subchorionic bleeding [3]. Pathogenesis is thought to be multifactorial including chromosomal abnormalities, the endocrinological and immunological causes being suspected to have an effect on the first trimester pregnancy loss [4]. There is insufficient evidence to support the use of progesterone for the prevention of pregnancy loss in pregnant women diagnosed with abortion imminens, and the bed rest does not appear to improve outcomes [5].

It is known that neutrophils, monocytes, lymphocytes and platelets in the blood play an active role in systemic inflammation. Rates calculated using complete blood count parameters have become popular in the diagnosis or prognosis of many diseases. In recent publications, the prognostic value of neutrophil lymphocyte ratio, platelet lymphocyte ratio and mean platelet volume has been investigated in spontaneous abortion [6], early threatened abortion [7], preeclampsia [8,9], HELLP syndrome [10] and in neonatal sepsis [11].

In this study, we aimed to investigate the relationship between miscarriage and the first trimester neutrophil lymphocyte ratio (NEU / LY), platelet lymphocyte ratio (PLT / LY), monocyte lymphocyte ratio (MO / LY), mean 
platelet volume lymphocyte ratio (MPV / LY), plateletcrit platelet ratio (PCT / PLT) and mean platelet volume (MPV).

\section{Materials and Methods}

The sample consisted of patients who were admitted to the Obstetrics and Gynecology Clinic of Umraniye Training and Research Hospital, Istanbul, Turkey between January 2016 and December 2019. Data regarding the diagnosis of abortus imminens and whose pregnancy ended before the 20th week was retrospectively obtained from the hospital records.

The following patients were excluded from the study: those with multiple pregnancies, those whose pregnancy started as multiple and then continued as a singleton, conceiving with in vitro fertilization, diagnosed as abortus incipient, a history of recurrent pregnancy loss or cervical insufficiency, a history of cervical leep or conization procedures, congenital uterine anomalies or uterine pathologies that disrupt the shape of the endometrial cavity, such as fibroids or polyps, diagnosed thrombophilia or using oral or parenteral anticoagulants and those who become pregnant while using an intrauterine device as a birth control method. In addition, patients who smoke or consume alcohol, with diabetes mellitus, adrenal gland, thyroid and parathyroid gland or liver disease, kidney dysfunction, oncological, infectious or hematological disease (including anemia), rheumatological or autoimmune disease, hyperlipidemia, atherosclerotic coronary artery disease and patients who received blood transfusion, intravenous iron replacement, or steroid use for any reason within the past year were also excluded from the study.

Patients diagnosed with abortus imminens received daily oral $2 \times 200 \mathrm{mg}$ progesterone and weekly intramuscular $250 \mathrm{mg}$ hydroxyprogesterone caproate. Despite this therapy, 39 patients had miscarriage before 20 weeks of gestation and constituted the study group. The control group consisted of 200 randomly selected patients with pregnancy follow-up and delivery in our hospital, and who did not have any pregestational or pregnancy related disease able to complicate their pregnancy.

Ultrasound examinations of the patients were performed with Hitachi Aloka Prosound F37 Ultrasound Machine. Complete blood count of the patients was performed on an automated hematology analyzer Mindray BC6800 machine. BMI of the patients was calculated by the following formula: Body Weight / Height Square (kg / $\mathrm{m} 2)$. NEU/LY was calculated by neutrophil count/ lymphocyte count, MO/LY was calculated by monocyte count/ lymphocyte count, PLT/LY was calculated by platelet count / lymphocyte count, MPV/LY was calculated by mean platelet volume/ lymphocyte count, PCT/PLT was calculated by plateletcrit/ platelet count.

\section{Statistics}

The data were analyzed using Statistical Package for the Social Sciences (SPSS) 25.0 package program (IBM Corp., Armonk, NY, USA). Descriptive statistical methods (mean, standard deviation, frequency) were used while evaluating the study data. The distribution of data was tested with the Kolmogorov Smirnov test. Parametric independent two samples t-test was used for normally distributed data, and non-parametric The Mann Whitney U-test was used for data that did not show normal distribution. A receiver operating characteristic (ROC) analysis was performed to determine the cut-off value for NLR for prediction of miscarriage. The significance threshold was evaluated at $\mathrm{p}<0.05$ levels for all values.

\section{Results}

The demographic characteristics and the first trimester hemogram results of the miscarriage and the control group were compared. Age and BMI of both groups were similar. There was no significant difference between the two groups in terms of basophil, neutrophil, eosinophil, lymphocyte, monocyte, platelets, RBC, hemoglobin, hematocrit, MCV, MCH, MCHC, RDW, MPV, Pct and PDW, while leukocyte and neutrophil counts was statistically significantly higher in the miscarriage group ( $\mathrm{p}=0,002, \mathrm{p}=0,001$, respectively) (Table 1 ). When both groups were compared in terms of NLR, MLR and PLR, NLR was statistically higher in the miscarriage group $(p=0,005)$, and PLR was also higher in the miscarriage group, but without reaching statistical significance $(\mathrm{p}=0,056)$. Both groups were similar in terms of MLR (Table 2).

ROC analysis was performed for miscarriage prediction of NLR values. According to the ROC analysis results, the area under the curve (AUC) value for NLR was found to be 0.642 . The threshold value that separates pregnancies with miscarriage by ongoing pregnancies was determined as 2.45 value, with $64,10 \%$ sensitivity and $63 \%$ specificity for NLR (Figure 1).

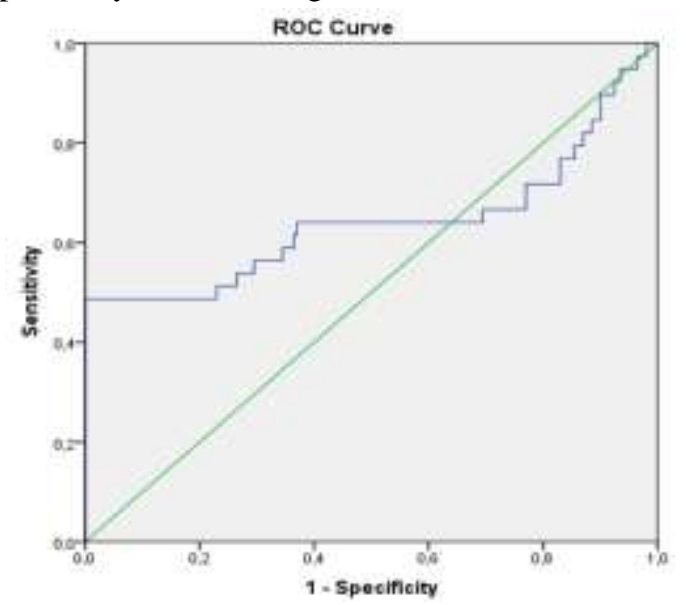

Figure1. Receiver operating characteristic curve analysis performed to examine the role of NLR in predicting miscarriage. (AUC $=0,642, p=0,005$ ) 
Table 1. Demographic characteristics and laboratory findings of the groups

\begin{tabular}{|c|c|c|c|}
\hline Variables & $\begin{array}{c}\text { Miscarriage group } \\
(\mathbf{n = 3 9 )} \\
\text { Mean } \pm \text { SD }\end{array}$ & $\begin{array}{c}\text { Control group } \\
(\mathbf{n = 2 0 0}) \\
\text { Mean } \pm \text { SD }\end{array}$ & p value \\
\hline Age & $29,26 \pm 5,96$ & $28,18 \pm 5,53$ & $0,273 *$ \\
\hline BMI & $23,68 \pm 1,27$ & $23,36 \pm 1,55$ & $0,111 *$ \\
\hline Leukocyte & $9296,67 \pm 2256,07$ & $8150,70 \pm 1837,60$ & $\mathbf{0 , 0 0 2} * *$ \\
\hline Neutrophil & $6326,41 \pm 2026,78$ & $5135,20 \pm 1407,22$ & $\mathbf{0 , 0 0 1} * *$ \\
\hline Basophil & $45,64 \pm 30,16$ & $43,65 \pm 60,97$ & $0,448 * *$ \\
\hline Eosinophil & $101,54 \pm 68,92$ & $125,40 \pm 99,69$ & $0,209 * *$ \\
\hline Lymphocyte & $2350,26 \pm 872,35$ & $2369,30 \pm 578,64$ & $0,517 * *$ \\
\hline Monocyte & $522,31 \pm 163,23$ & $484,60 \pm 164,74$ & $0,108 * *$ \\
\hline Platelet & $266794,87 \pm 54403,07$ & $255195,00 \pm 58105,41$ & $0,251 *$ \\
\hline RBC & $4,23 \pm 0,27$ & $4,33 \pm 0,31$ & $0,081 *$ \\
\hline Hemoglobin & $12,35 \pm 0,80$ & $12,47 \pm 0,81$ & $0,365 *$ \\
\hline Hematocrit & $36,71 \pm 2,38$ & $37,12 \pm 2,37$ & $0,320 *$ \\
\hline MCV & $88,76 \pm 4,05$ & $85,96 \pm 5,16$ & $0,364 *$ \\
\hline MCH & $29,17 \pm 1,67$ & $28,90 \pm 1,96$ & $0,419 *$ \\
\hline MCHC & $33,49 \pm 1,13$ & $33,57 \pm 1,00$ & $0,646 *$ \\
\hline RDW & $14,37 \pm 1,45$ & $14,56 \pm 1,69$ & $0,690 * *$ \\
\hline MPV & $8,96 \pm 1,67$ & $9,14 \pm 1,55$ & $0,511 *$ \\
\hline Pct & $0,24 \pm 0,06$ & $0,23 \pm 0,06$ & $0,601 * *$ \\
\hline PDW & $16,75 \pm 1,42$ & $16,80 \pm 1,15$ & $0,377 * *$ \\
\hline
\end{tabular}

* Intependent $\mathrm{T}$ test , ** Mann Whitney U test

Table 2. Comparison of the groups in terms of NLR, MLR and PLR.

\begin{tabular}{|c|c|c|c|}
\hline & $\begin{array}{c}\text { Miscarriage group } \\
(\mathbf{n}=\mathbf{3 9}) \\
\text { Mean } \pm \text { SD }\end{array}$ & $\begin{array}{c}\text { Control group } \\
(\mathbf{n = 2 0 0}) \\
\text { Mean } \pm \text { SD }\end{array}$ & p value \\
\hline NLR & $3,10 \pm 1,72$ & $2,22 \pm 0,52$ & $\mathbf{0 , 0 0 5} * *$ \\
\hline MLR & $0,24 \pm 0,10$ & $0,21 \pm 0,07$ & $0,134 * *$ \\
\hline PLR & $125,81 \pm 42,94$ & $111,74 \pm 29,45$ & $0,056 *$ \\
\hline
\end{tabular}

NLR: neutrophil to lymphocyte ratio, MLR: monocyte to lymphocyte ratio, PLR: platelet to lymphocyte ratio. * Intependent T test, ** Mann Whitney U test

\section{Discussions}

The implantation of the embryo depends on the harmonious interaction between the placenta and the endometrium. Abnormal physiological regulation of immunity, increased decidual or systemic inflammation during pregnancy may be one of the causes of miscarriage. Systemic inflammation can be measured using a variety of biochemical and hematological markers. The ratio of blood cell subtypes such as NLR, MLR and PLR may have prognostic significance for inflammation-related diseases. In particular, NLR has been investigated in many diseases in obstetrics and it has been shown to be associated with hyperemesis gravidarum [12], intrahepatic pregnancy cholestasis [13] and preeclampsia [8,9].

In a study conducted with patients diagnosed with abortus imminens in the first trimester, the NLR was found to be statistically significantly higher in the group that resulted in abortion, comparative to the group with ongoing pregnancy after 24 gestational week ( $p<0.001)$ [14]. Similarly, Bas et al found that the NLR was significantly higher in patients with abortion in the first and second trimesters, versus the control group that had a live birth $(p<0,0001)[6]$. On the contrary, according to another study 
investigating the relationship of NLR with miscarriage, no statistically significant difference was found in terms of NLR related to the healthy control group, the group with the threatened abortion and the group with early pregnancy loss $(p=0,19)$ [15]. Similarly, Christoforaki et al. stated that NLR did not differ between pregnant women who had live births and those who had miscarriage ( $p=0.167$ ). Even so, they noted that NLR values above 5.8 were encountered only in the miscarriage group [16].

In this study, we found that NLR in the miscarriage group was significantly higher than the control group with a live birth $(\mathrm{p}=0,005)$. ROC analysis was performed for the value of NLR for predicting miscarriage. The optimal cutoff value of the first trimester NLR to predict miscarriage was 2,45 with a sensitivity of $64,1 \%$ and a specificity of $63 \%$. We attributed the different results of the studies examining the relationship between NLR and miscarriage to several parameters, such as the difference in the number of patients included in the studies, the differences in the inclusion and exclusion criteria and differences in the definition of the control groups. In addition, in some studies, it was not clearly stated whether the group with miscarriage received progesterone treatment before.

In the study published by Wang et al in 2020, they compared the missed abortion group with the control group with medical abortion in early pregnancy with a healthy, viable intrauterine fetus and no prior missed abortion history. In addition to NLR, they found that MLR was significantly lower in the missed abortion group compared to the control group ( $\mathrm{p}=0.000, \mathrm{p}=0.003$, respectively) [17]. Unlike this study, we did not observe any significant difference in terms of first trimester MLR between the miscarriage group and the live birth control group in our study $(\mathrm{p}=0,134)$.

Many studies in the literature also examined the relationship between PLR and miscarriage. In a study published in 2020, PLR was found significantly higher in the early pregnancy loss group than the group with threatened abortion and the healthy controls ( $p<0.001)$ [15]. Biyik et al. also found PLR significantly higher in the missed abortion group compared to the healthy pregnant group ( $p=0.01$ ) [18]. Consistent with these data, in the study conducted by Onat et al., the PLR rate was found to be significantly higher in the abortion group compared to the control group $(p=0,032)$ [19]. In a different study, PLR was found to be similar in the miscarriage group and the control group whose pregnancy continued after 24 weeks. ( $p=0.071$ ) [14]. On the contrary, in the study conducted by Bas et al., PLR was significantly lower in the group with miscarriage in the first and second trimesters compared to the control group with live births $(p=0.03)$ [6]. In the present study, we found a higher PLR in the miscarriage group compared to the control group that had a live birth, but this did not reach the statistical significance $(\mathrm{p}=0.056)$.

MPV is another hemogram parameter investigated in miscarriage. The authors thought that changes in platelet activity and volume during pregnancy might be associated with miscarriage. Larger platelets are thought to have granules with more mediators, being perhaps more active. Bas et al stated that the MVP was significantly lower in patients with abortion in the first and second trimesters compared to the control group that had a live birth ( $p<0,0001$ ) [6]. Also, in a study published in 2020, MPV was found significantly lower in the early pregnancy loss group than the healthy controls $(p=0.001)$ [15]. Consistent with these, Kaplanoglu et al. found in their study that the MPV level was significantly lower in the group with miscarriage compared to the control group ( $p<0.001$ ) [20]. In our study, we also found that the MPV value was lower in the miscarriage group compared to the control group that had a live birth, but this value was not statistically significant. While MPV value was expected to be higher in pregnancies resulting in miscarriage compared to the control group, the opposite data was found in other studies. The authors explained this situation through the fact that platelets with higher activity (larger platelets) due to inflammation and bleeding may migrate to the deciduoplacental unit earlier in the gestational weeks, and this may cause a decrease in MPV in the peripheral circulation of patients in the miscarriage group [15,20].

The study has some limitations that should be mentioned. It was designed retrospectively and the number of cases included was relatively low. In addition, the lack of use of other proven markers of inflammation is one of the points that should be kept in mind and should be regulated in further researches.

\section{Conclusions}

In conclusion, we found that high NLR levels obtained from the hemogram results in the first trimester were associated with miscarriage. We think that NLR can be useful in predicting pregnancy evolution because of its cost-effectiveness and non-invasiveness. However, these results should be supported by large-scale and better designed prospective studies.

\section{Conflict of interest disclosure}

There are no known conflicts of interest in the publication of this article. The manuscript was read and approved by all authors.

\section{Compliance with ethical standards}

Any aspect of the work covered in this manuscript has been conducted with the ethical approval of all relevant bodies and that such approvals are acknowledged within the manuscript. Ethical approval of the study was obtained from the local institutional ethics committee (B.10.1.TKH.4.34.H.GP.0.01/7) and the study was conducted in accordance with the Declaration of Helsinki. 


\section{References}

1. Hendriks E, MacNaughton H, MacKenzie MC. First Trimester Bleeding: Evaluation and Management. Am Fam Physician. 2019;99(3):166-174.

2. Evrenos AN, Cakir Gungor AN, Gulerman C, Cosar E. Obstetric outcomes of patients with abortus imminens in the first trimester. Arch Gynecol Obstet. 2014;289(3):499-504. doi: 10.1007/s00404-013-2979-5

3. Poulose T, Richardson R, Ewings P, Fox R. Probability of early pregnancy loss in women with vaginal bleeding and a singleton live fetus at ultrasound scan. J Obstet Gynaecol. 2006;26(8):782-4. doi: 10.1080/01443610600984412

4. Alijotas-Reig J, Garrido-Gimenez C. Current concepts and new trends in the diagnosis and management of recurrent miscarriage. Obstet Gynecol Surv. 2013;68(6):445-66. doi: 10.1097/OGX.0b013e31828aca19

5. The American College of Obstetricians and Gynecologists Practice Bulletin no. 150. Early pregnancy loss. Obstet Gynecol. 2015;125(5):12581267. doi: 10.1097/01.AOG.0000465191.27155.25

6. Bas FY, Tola EN, Sak S, Cankaya BA. The role of complete blood inflammation markers in the prediction of spontaneous abortion. Pak J Med Sci. 2018;34(6): 1381-1385. doi: 10.12669/pjms.346.15939

7. Feng QT, Chen C, Yu QY, Chen SY, Huang X, Zhong YL, Luo SP, Gao J. The benefits of higher LMR for early threatened abortion: A retrospective cohort study. PLoS One. 2020;15(4):e0231642. doi: 10.1371/journal.pone.0231642

8. Gogoi P, Sinha P, Gupta B, Firmal P, Rajaram S. Neutrophil-to-lymphocyte ratio and platelet indices in pre-eclampsia. Int J Gynaecol Obstet. 2019;144(1):1620. doi: 10.1002/ijgo.12701

9. Wang J, Zhu QW, Cheng XY, Liu JY, Zhang LL, Tao YM, Cui YB, Wei Y. Assessment efficacy of neutrophil-lymphocyte ratio and monocytelymphocyte ratio in preeclampsia. J Reprod Immunol. 2019;132:29-34. doi: 10.1016/j.jri.2019.02.001

10. Sisti G, Faraci A, Silva J, Upadhyay R. Neutrophil-toLymphocyte Ratio, Platelet-to-Lymphocyte Ratio and Complete Blood Count Components in the First Trimester Do Not Predict HELLP Syndrome. Medicina (Kaunas). 2019;55(6):219. doi: 10.3390/medicina55060219
11. Can E, Hamilcikan Ş, Can C. The Value of Neutrophil to Lymphocyte Ratio and Platelet to Lymphocyte Ratio for Detecting Early-onset Neonatal Sepsis. J Pediatr Hematol Oncol. 2018;40(4):e229-e232. doi: 10.1097/MPH.0000000000001059

12. Caglayan EK, Engin-Ustun Y, Gocmen AY, Sarı N, Seckin L, Kara M, Polat MF. Is there any relationship between serum sirtuin-1 level and neutrophillymphocyte ratio in hyperemesis gravidarum? J Perinat Med. 2016;44(3):315-20. doi: 10.1515/jpm-2015-0178

13. Yayla Abide Ç, Vural F, Kılıççı Ç, Bostancı Ergen E, Yenidede İ, Eser A, Pekin O. Can we predict severity of intrahepatic cholestasis of pregnancy using inflammatory markers? Turk J Obstet Gynecol. 2017;14(3):160-165. doi: 10.4274/tjod.67674

14. Kan E. The effect of systemic inflammation markers on predicting pregnancy results in patients admitted to emergency department with threatened miscarriage. Ank Med J. 2019;19(2):337-343. doi: 10.17098/amj.576455

15. Ata N, Kulhan M, Kulhan NG, Turkler C. Can neutrophillymphocyte and platelet-lymphocyte ratios predict threatened abortion and early pregnancy loss? Ginekol Pol. 2020;91(4):210-215. doi: 10.5603/GP.2020.0042

16. Christoforaki V, Zafeiriou Z, Daskalakis G, Katasos T, Siristatidis C. First trimester neutrophil to lymphocyte ratio (NLR) and pregnancy outcome. J Obstet Gynaecol. 2020;40(1):59-64. doi: 10.1080/01443615.2019.1606171

17. Wang Q, Liu F, Zhao Y, Cui B, Ban Y. Can neutrophilto-lymphocyte and monocyte-to-lymphocyte ratios be useful markers for predicting missed abortion in the first trimester of pregnancy? J Obstet Gynaecol Res. 2020;46(9):1702-1710. doi: 10.1111/jog.14349

18. Biyik I, Albayrak M, Keskin F. Platelet to Lymphocyte Ratio and Neutrophil to Lymphocyte Ratio in Missed Abortion. Rev Bras Ginecol Obstet. 2020;42(5):235239. doi: 10.1055/s-0040-1709693

19. Onat T, Aydoğan Kirmizi D, DemiR ÇaltekiN M, Başer E, Yalvaç ES. Can hematologic inflammation markers be the indicator of early pregnancy loss? J Surg Med. 2020;4(11):952-5.

20. Kaplanoglu M, Yuce T, Bulbul M. Decreased mean platelet volume is associated with the developing stage of fetoplacental unit in spontaneous abortion. Int J Clin Exp Med. 2015 Jul 15;8(7):11301-6. 